\title{
Sobre as origens das Leis de Fresnel
}

\author{
On the origins of Fresnel's Laws
}

\author{
M. C. de Lima*10, Lucas Silva \\ ${ }^{1}$ Universidade Federal do Pará, Faculdade de Física, Rua Augusto Corrêa 1, Belém, PA, Brasil
}

Recebido em 14 de Outubro, 2018. Revisado em 14 de Fevereiro, 2019. Aceito em 20 de Fevereiro, 2019.

\begin{abstract}
Revisitamos o desenvolvimento da teoria ondulatória da luz, no período que se seguiu à hipótese de Fresnel de que o éter luminífero possuia natureza similar a de um sólido elástico. Examinamos como em tal teoria elástica da luz se produziu um legado presente, ainda hoje, nas entrelinhas do eletromagnetismo clássico, tomando as leis de Fresnel como baliza. Procuramos também indicar como as limitações desta abordagem contribuíram para criar um ambiente favorável à apropriação perpetrada por Maxwell, em favor de uma teoria eletromagnética da luz. Palavras-chave: Teoria elástica da Luz, éter luminífero, Teoria eletromagnética da luz.
\end{abstract}

We revisit the development of the wave theory of light in the period following Fresnel's hypothesis that the luminiferous ether was similar in nature to an elastic solid. We have examined how, in such elastic theory of light, a legacy was produced wich is present, still today, in the body of classical electromagnetism, taking the laws of Fresnel as beacon. We also seek to indicate how the limitations of this approach contributed to create an environment conducive to the appropriation perpetrated by Maxwell in favor of an electromagnetic theory of light. Keywords: Elastic theory of light, luminiferous ether, Electromagnetic theory of light.

\section{Introdução}

Como é sabido [1] 3 , a intensidade relativa de um feixe de luz refletido, na interface plana que separa dois meios isotrópicos, $I_{R} / I$, depende do tipo de polarização do feixe incidente, estando seus valores sintetizados nas Leis de Fresnel

$$
\begin{aligned}
& \left(\frac{I_{R}}{I}\right)_{\perp}=\left[\frac{\operatorname{sen}\left(\theta_{i}-\theta_{t}\right)}{\operatorname{sen}\left(\theta_{i}+\theta_{t}\right)}\right]^{2}, \\
& \left(\frac{I_{R}}{I}\right)_{\|}=\left[\frac{\tan \left(\theta_{i}-\theta_{t}\right)}{\tan \left(\theta_{i}+\theta_{t}\right)}\right]^{2} .
\end{aligned}
$$

Nestas expressões o subscrito $\perp$ se refere à luz plano polarizada perpendicularmente ao plano de incidência, enquanto que o caso denotado por $\|$ é aquele em que a polarização do feixe incidente é paralela ao plano de incidência. Os $\left(\theta_{i}, \theta_{t}\right)$ são os ângulos de incidência e de refração, como definidos usualmente, isto é, os ângulos agudos formados entre a direção do correspondente raio e a direção normal à interface.

Apresentados em textos didáticos atuais como consequência das condições de contorno impostas ao campo eletromagnético pelas equações de Maxwell, no contexto da teoria eletromagnética da luz, os coeficientes de refle$x \tilde{a} o$ de Fresnel,

$$
R_{\perp}=\frac{\operatorname{sen}\left(\theta_{i}-\theta_{t}\right)}{\operatorname{sen}\left(\theta_{i}+\theta_{t}\right)}
$$

*Endereço de correspondência: mclima@ufpa.br

$$
R_{\|}=\frac{\tan \left(\theta_{i}-\theta_{t}\right)}{\tan \left(\theta_{i}+\theta_{t}\right)},
$$

podem ser aí encarados como remanescentes de uma outra época, agora superada pelos desenvolvimentos ulteriores. Relações que remontam ao tempo em que a teoria eletromagnética sequer havia sido formulada. Época na qual os fenômenos da óptica eram discutidos com base na premissa da existência de um éter luminífero e vistos como uma das faces observáveis dos processos mecânicos que nele ocorriam.

Neste trabalho revisitamos o desenvolvimento da teoria ondulatória da luz no período que se seguiu à hipótese de Fresnel, de que o éter luminífero tinha natureza similar à de um sólido elástico, nos anos de 1820, recapitulando o debate que se seguiu, até o entorno do ano de 1888, quando Hertz produziu ondas eletromagnéticas em laboratório. Tomando por baliza as leis de Fresnel, (1) e (2), e examinando os desenvolvimentos teóricos que buscavam encontrar a justificativa das mesmas, podemos vislumbrar como em tal teoria elástica da luz se produziu um legado presente, ainda hoje, nas linhas ou entrelinhas da teoria eletromagnética clássica. Procuramos também indicar como as limitações desta abordagem propiciaram o ambiente favorável à "apropriação da luz", perpetrada por Maxwell, que passou a advogar uma teoria eletromagnética da luz. No entanto, a teoria eletromagnética de Maxwell, hoje consagrada dentro dos limites de uma descrição não-quântica da natureza, foi vista àquela altura tanto como avanço parcial quanto retrocesso. Procuramos aqui fornecer indícios do porquê. 


\section{Limitações do éter fluido}

A premissa de que não existe lugar vazio no universo acompanhou as especulações científicas em várias épocas, no curso da civilização ocidental, desde o alvorecer das ciências naturais no século $V$ a.C., na Grécia. Aristóteles considerou que os espaços cósmicos aparentemente vazios estavam, na verdade, preenchidos pelo quinto elemento, ou quintessência, em sua versão da doutrina dos elementos Ar, Terra, Fogo e Água.

Com o advento da ciência moderna Descartes também concebeu o espaço como um plenum que definia a sua extensão [4], distinguindo as qualidades da matéria por suas propriedades relativas à luz, em três tipos: a matéria luminosa de que é feito o sol e as demais estrelas, a matéria transparente do espaço interplanetário e a matéria opaca dos planetas e cometas. A luz seria uma pressão comunicada instantaneamente 1 através da matéria do segundo tipo. Esta velocidade infinita de transmissão da pressão não o impediu de deduzir a lei da refração 2 em seu La Dioptrique, de 1638. Surgiu assim, através da matéria do segundo tipo de Descartes, a noção moderna de um éter que a tudo permeia. Descartes viu no éter a explicação da ação mútua entre corpos que não estão em contato aparente [5]. Neste contexto, o éter seria sutil o suficiente para nã̃o nos fornecer evidência direta de sua onipresença. Porém, reativo às alterações de seu estado físico, o suficiente, para gerar fenômenos observáveis. Fenômenos de intensidade suficiente para causar modificações nos estados da matéria usual, ponderável, cuja existência é evidente.

Uma vez apontada como evidência de algum tipo de distúrbio propagado através do éter, esta visão sobre a natureza da luz logo foi endossada por Hooke [6], em seu Micrographia, de 1665, e por Huygens [7], em seu Traité de la lumière, de 1690, quando atribuiu elasticidade ao meio etéreo, análoga, porém de natureza distinta, àquela do ar. Apontou a necessidade dele possuir elevada resistência à compressão, de modo a propagar distúrbios tão rapidamente quanto a luz. Argumentou em favor de sua penetrabilidade tanto através dos corpos transparentes quanto opacos. Foi também neste tratado que estabeleceu o princípio que ainda hoje leva seu nome. Em seus estudos sobre a propagação da luz em meios cristalinos, abordou o fenômeno da dupla refração 3 quando desenvol-

${ }^{1}$ A evidência experimental de que a luz afinal se propaga com velocidade finita surgiu em 1676, quando Olaf (Olaus) Roemer, no Observatório de Paris, descobriu que os eclipses das luas de Júpiter se atrasam, sucessivamente, enquanto Júpiter se afasta relativamente à Terra, e se adiantam quando Júpiter se aproxima relativamente a Terra, enquanto trabalhava nas tabelas das efemérides. O atraso na observação dos eclipses poderia ser explicado pela hipótese de que a luz não se propaga instantaneamente, o que possibilitou a Roemer medir a velocidade da luz obtendo: $2,3 \times 10^{8} \mathrm{~m} / \mathrm{s}$ (Journal des Sçavans, pp. 276-279, 7 (1676), e Phil. Trans. Roy. Soc., No. 136, (1677)).

${ }^{2}$ Willebrord Snell a havia descoberto empiricamente entre 1621 e 1625, embora Descartes não o cite.

${ }^{3}$ A dupla refração, ou birrefringência, fora descoberta por Erasmus Bartholin (Bartolinus), em 1669, ao notar a dupla imagem formada a partir de um único objeto, quando visto através do espato da Islândia, um cristal de calcita. veu uma versão modificada de seu princípio; admitindo que um ponto na frente de onda incidente gera duas novas frentes de onda: uma esférica e outra elipsoidal. A primeira associada ao raio ordinário e a segunda ao raio extraordinário, nomenclaturas por ele introduzidas. Afinal, não pôde oferecer uma explicação conclusiva dos vários aspectos desse fenômeno. Huygens também não encarou a luz como um movimento ondulatório periódico, mas em pulsos.

Com a gradual aceitação dos pressupostos estabelecidos por Newton nos Principia, publicado em 1687, no qual figurava a ação instantânea à distância, bem como o espaço vazio e inerte, a ideia da luz como distúrbio do meio etéreo conheceu um relativo ostracismo durante o século XVIII. Também contribuiu para isso o Opticks de Newton, cuja primeira edição surgiu em 1704, que foi em grande medida interpretado como uma visão de que a luz tinha uma natureza corpuscular. Em ambas as questões, a das partículas luminosas e da ação instantânea à distância, Newton parece não ter sido tão enfático [8], na defesa das opiniões a ele atribuídas, quanto o foram os seguidores de uma tradição de pensamento proclamada "newtoniana". Distinguindo-se porém da tendência de abandono do éter podemos citar a obra óptica de Euler e a mémoire de Johann II Bernoulli, "Pesquisas físicas e geométricas sobre a questão: Como se dá a propagação da luz" [9], que arrematou o prêmio da Academia de Paris de 1736.

Adentrando o século XIX, Thomas Young trouxe vigor renovado ao programa da teoria ondulatória da luz, com seus experimentos relacionados ao fenômeno que batizou de interferência. Em sua comunicação à Royal Society de 24 de novembro de 1803 [10], apresentou os comprimentos de onda associados à luz visível, entre 400 e 700 nanômetros, o que fornecia não somente evidência do caráter ondulatório da luz, como também da natureza periódica deste movimento.

Na França revolucionária adeptos da chamada teoria corpuscular da luz dominavam a cena. Em 1810 a Academia de Ciências de Paris premiou a memória de étienne Malus, acerca da dupla refração na óptica cristalina ${ }^{4}$ Aí Malus introduziu a terminologia polarização da luz para designar as qualidades distintas do raio ordinário e extraordinário na dupla refração. Malus também descobrira pouco antes, em 1808, a polarização por reflexão de um feixe de luz. No contexto de sua memória, entretanto, o termo polarização era uma qualidade das partículas de luz, que se deformavam analogamente a uma esfera que pode deformar-se em um elipsóide prolato ou oblato. Na Escócia, David Brewster tomou conhecimento da descoberta de Malus no verão de 1811. Explorou experimentalmente as características gerais da polarização por reflexão. Observou o desaparecimento do feixe refletido quando o feixe incidente estava polarizado em

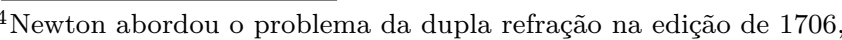
em latim, de seu Óptica, quando então propôs que os raios luminosos possuíam "lados" e, por isso, podiam apresentar propriedades ópticas diferentes nas distintas direções do plano normal à direção de propagação (isto é, um plano como aquele que chamaríamos de frente de onda na visão ondulatória).
} 
uma das duas possíveis polarizações, e na situação em que os ângulos de incidência e de reflexão eram complementares. Esta então chamada lei de Brewster será justificada por Fresnel mais adiante, com base na lei das tangentes. Estabeleceu também a relação entre o ângulo no o qual se dá a polarização por reflexão e o índice de refração do meio, ângulo este que hoje leva seu nome [11]. Nos seus estudos de óptica cristalina, Brewster revelou a existência dos cristais biaxiais [12], nos quais haviam dois eixos segundo os quais a dupla refração não ocorria. No espato da Islândia havia somente um eixo sobre o qual a dupla refração era suprimida, e este ficou assim chamado uniaxial. Os cristais biaxiais punham em xeque a construção original de Huygens para explicar a dupla refração e, consequentemente, o programa da teoria ondulatória da luz. Na verdade, como ficaria evidente em desenvolvimentos posteriores, somente era um impasse para a teoria ondulatória na medida em que a luz era encarada como onda acústica no éter, isto é, longitudinal, e o éter, por sua vez, um fluido, isto é, incapaz de propagar ondas cisalhantes.

É então que entra em cena Augustin-Jean Fresnel (1788-1827), que ganhou o prêmio da Academia de Paris, de 1818, com sua "Memória sobre a difração da luz" [13]. Sua "mémoire", baseada na hipótese ondulatória da luz, finalmente trazia ao centro da arena o programa iniciado por Huygens. Temos aí a primeira formulação matemática da teoria ondulatória da luz, havendo Fresnel colocado o princípio de Huygens em bases matemáticas precisas (sobre o rico debate que levou à emergência da teoria ondulatória da luz, neste período, indicamos [14]). A previsão da existência de um ponto luminoso no centro da sombra geométrica de um disco e a confirmação experimental de sua existência promoveu a conversão de Poisson, um dos revisores da mémoire de Fresnel, à teoria ondulatória da luz. Mesmo Newton defendera textualmente que a inflexão dos raios luminosos (isto é, a difração) sempre ocorria para fora da região da sombra geométrica, nunca para dentro 15$]$. Mas foi nos seus estudos de óptica em meios cristalinos que Fresnel foi levado ao estudo das propriedades da luz polarizada. Tentou em vão, juntamente com Francois Arago, produzir interferência entre dois raios de luz polarizados em planos perpendiculares entre si. Numa famosa carta de Young a Arago, de 17 de janeiro de 1817, Young sentenciou: as vibrações da luz são transversais à direção de propagação e a razão de haverem duas polarizações independentes, uma perpendicular a outra, deve-se à existência de dois planos, perpendiculares um ao outro, e perpendiculares à direção de propagação, sobre os quais este movimento vibratório pode ser decomposto. O artigo de Fresnel e Arago, descrevendo tais resultados, somente surgiu em 1819, intitulado "Sobre a ação dos raios de luz polarizados um sobre o outro" [16], onde os experimentos são discutidos sem, no entanto, haver comprometimento quanto à natureza, longitudinal ou transversal, das vibrações luminosas. Arago preferiu não se comprometer acerca da hipótese da natureza transversal, cabendo a Fresnel dar o passo seguinte [17].
Nasceu aí uma das importantes vertentes da física teórica do século XIX, a qual podemos pensar como um retorno vigoroso à física do plenum. Nela se buscou imputar ao éter (ou aos éteres) todas as ações físicas que se lançam através do espaço. Nela reuniu-se num só corpo teórico não somente a propagação da luz e os correspondentes fenômenos ópticos, mas também a radiação térmica, a ação magnética, elétrica e eletromagnética. Sua culminância e gradual esgotamento nos deixou como legado a teoria eletromagnética da luz de Maxwell. Antes porém que a ideia do campo se consolidasse como um ente físico desprovido de substrato, é que se travou o embate que pretendemos aqui rememorar.

\section{As Leis de Fresnel por Fresnel}

O ano de 1821 marca a adesão de Fresnel à concepção de que o éter luminífero não podia ser um fluido. Tornava-se assim o fundador da teoria ondulatória da luz baseada em um éter do tipo sólido-elástico [18. Em seu "Sobre o cálculo das matizes que a polarizaçã̃o desenvolve em lâminas cristalinas" [19] deixa claro sua aceitação de que o éter luminífero podia propagar deformações transversais, isto é, ondas de cisalhamento, fazendo considerações mecânicas sobre a sua estrutura. Originalmente Fresnel introduzira, juntamente com Ampère, a ideia de que a onda associada à luz polarizada poderia ainda ter um componente longitudinal. Já agora argumentava em favor do caráter exclusivamente transverso dela. A ausência de ondas longitudinais foi por ele justificada pela suposição de que a resistência do éter luminífero à compressão era muito mais forte que sua resistência à distorção. Disse ele ( 19], p.633):

"Permanece para mim a ser explicado, como pode que as moléculas [do éter] não sofram oscilações significativas que não aquelas na própria superfície das ondas, perpendiculares aos raios. Bastaria para isso supor uma lei de repulsão entre as moléculas [do éter] tal que a força que se opõe à aproximação mútua de duas lâminas de fluido é muito maior que aquela que se opõe ao deslizamento de uma com relação à outra."

Deste modo, defendeu que a velocidade das ondas longitudinais seria tão grande quando comparada à da luz, entendida como onda de cisalhamento, que, na prática, um equilíbrio de pressão seria mantido perpetuamente.

Fresnel considerou que além de transversais à direção de propagação, as deformações do meio etéreo $\left(\vec{e}_{p}\right)$, em uma onda polarizada, ocorriam perpendicularmente ao plano de polarização, o que pode ser escrito em linguagem anacrônica como,

$$
\vec{e}_{p} \propto \hat{n}_{p}
$$

em que $\hat{n}_{p}$ é o versor normal que identifica o particular plano de polarização, $\vec{e}_{p}$ o vetor deformação elástica, associado ao particular modo de polarização, no ponto em questão. Seguindo sugestão de Young, também assumiu que as mudanças na velocidade de propagação $(c)$, em 
meios transparentes distintos, eram devidas às variações de densidade $(\rho)$ do éter luminífero, na presença destes meios, na forma

$$
c \propto \sqrt{\frac{1}{\rho}}
$$

mantendo-se invariantes as propriedades elásticas do meio.

Ambas as premissas estarão suscetíveis à variações na literatura subsequente. Com relação à primeira, autores posteriores usarão tanto a hipótese de Fresnel quanto a hipótese de que a vibração ocorre no próprio plano de polarização. É uma questão que se revelará afinal supérflua, à época em que Heinrich Hertz demonstrar a existência das ondas eletromagnéticas, em 1888. Nas palavras de Hertz ( 20], p.178),

"[...]a questão de qual dos dois planos é aquele em que ocorre a oscilação do raio não pode ser respondida; a menos que especifiquemos se a questão refere-se à oscilação elétrica ou magnética.[...] esta consideração é a razão pela qual a antiga disputa óptica jamais foi decidida."

No tocante à velocidade de propagação, pode-se igualmente atribuir tal mudança de velocidade à mudança das características elásticas do éter luminífero, quando da passagem de uma substância transparente à outra, o que também será explorado por investigadores subsequentes.

Em 19 de novembro de 1821 Fresnel apresentou, perante a Academia de Paris, sua "Primeira memória sobre a dupla refração" em que explorou tanto a dupla refração sobre cristais uniaxiais (Bartholinus, 1669) quanto biaxiais (Brewster, 1815), introduzindo as superfícies de onda de Fresnel, o hoje chamado elipsóide de índices, das quais eram obtidas as velocidades de propagação do raio ordinário e extraordinário, como raízes destas formas quadráticas [21]. Na descrição da dupla refração, Huygens empregara originalmente o método que consistia em considerar duas frentes de onda uma esférica e outra elipsoidal (em cristais uniaxiais). A descoberta dos cristais biaxiais comprometera o esquema original de Huygens, mas apenas parcialmente, na medida em que a luz era tomada como longitudinal naquele contexto. Fresnel propunha agora uma versão modificada do método de Huygens que o recuperava, como caso particular, quando o cristal era uniaxial, isto é, quando o elipsóide de índices de refração é um elipsóide de revolução. Esta memória de Fresnel, no entanto, somente será publicada em suas "Oeuvres", muito depois de sua morte, em 1868.

Tanto na obra de Fresnel quanto na daqueles que o sucederam, as pesquisas sobre a óptica dos meios cristalinos seguiu de forma paralela a dos meios isotrópicos. No presente artigo, entretanto, iremos nos limitar aos meios isotrópicos, mencionando os desenvolvimentos em óptica cristalina apenas superficialmente quando se faz necessário, para a narrativa histórica. Para além disto, a teoria elástica da luz em meios cristalinos foge ao escopo do presente artigo.
As hoje chamadas leis de Fresnel, dos senos (1) e das tangentes (2), surgiram na memória de Fresnel "Sobre a lei das modificações que a reflexão imprime sobre a luz polarizada" [22], de 1823, quando ele exibiu os coeficientes de reflexão, (3) e (4), associados a cada modo de polarização. Estes viriam a se tornar, por assim dizer, o "Santo Graal" das teorias elásticas. Isto é, qualquer teoria elástica da luz deveria necessariamente revelar o caminho até eles.

Embora as Leis de Fresnel estivessem em bom acordo com a experiência, a dedução de Fresnel não podia ser considerada consistente com os princípios da mecânica. Por intensidade ( $I$ ) Fresnel entendia a magnitude da velocidade vibracional das partículas da porção etérea em consideração. Assim, para deduzir (3) e (4) Fresnel impôs ad hoc que em sua passagem através de uma interface $(\Sigma)$, separadora de dois meios homogêneos e isotrópicos, houvesse conservação da vis viva. Em termos anacrôni$\cos$, isto equivale à conservação da energia cinética $\left(E_{c}\right)$ vibracional da porção do meio etéreo entre duas frentes de onda sucessivas, a saber, aquela que acaba de atravessar a interface e aquela que acaba de chegar à interface. Matematicamente,

$$
E_{c(m e i o 1)}=E_{c(m e i o 2)} .
$$

Além desta condição impôs também a continuidade do componente tangencial do vetor deformação elástica de um elemento substancial do éter luminífero $(\vec{e})$ sobre a interface. Se denotarmos por $[f]_{\Sigma}$, a descontinuidade de uma certa grandeza $f$ através da superfície de separação, $\Sigma$, então, em notação atual, a condição de continuidade do componente tangencial de $\vec{e}$ poderia ser expressa como

$$
[\hat{n} \times(\vec{e} \times \hat{n})]_{\Sigma}=0 .
$$

Destes pressupostos resultam (3) e (4), porém, igualmente se conclui que o componente normal $\left(e_{n}\right)$ da deformação elástica do éter sofre uma descontinuidade sobre a interface, isto é,

$$
\left[e_{n}\right]_{\Sigma}=[\vec{e} \cdot \hat{n}]_{\Sigma} \neq 0 .
$$

Tal condição é fisicamente improvável, na medida em que isto implica na aceitação de que o meio luminífero sofre um "rasgo" perpendicularmente à interface. A restauração da continuidade de $e_{n}$ podia ser feita reintroduzindo ondas longitudinais (acústicas) no éter, ainda que não constituindo a luz, já que a luz seria o modo cisalhante. Introduzi-las porém implica em reconsiderar o conteúdo de energia cinética vibracional do meio etéreo e todo o argumento original usado na dedução de (3) e (4) sucumbia.

Apesar de sua monumental contribuição para o restabelecimento do programa de uma óptica ondulatória, Fresnel deixaria aberto a resolução deste problema, aparentemente simples, da reflexão da luz polarizada, em meios isotrópicos; dentro do paradigma de ser a luz uma onda cisalhante no éter tipo sólido elástico. Honrado na França desde o prêmio da academia pela sua mémoire 
sobre a difração, em 1819, eleito membro da Académie Royale des Sciences de Paris em 1823, reconhecido na Inglaterra e agraciado com a medalha Rumford da Royal Society, em 1827, Fresnel não viveria para tomar parte nos desdobramentos do problema da reflexão/refração. Veio a falecer vitimado pela tuberculose, aos 39 anos, neste mesmo ano de 1827.

Impor as condições de contorno corretas sobre a interface refletora/refratora de modo consistentes com os princípios da mecânica, bem como atribuir ao éter luminífero as propriedades elásticas apropriadas, de modo a evitar o modo acústico, ou mesmo esclarecer o papel que ele desempenha, se mostrará afinal, de certa forma, uma empreitada cuja solução foi, à luz da história, insatisfatória.

\section{O desenvolvimento da teoria da elasticidade}

Enquanto Fresnel cogitava que o éter luminífero pudesse possuir propriedades elásticas, o modo de se estender a Lei de Hooke ao contínuo, no contexto da mecânica, ganhava vigor renovado. Em 1821 Louis-Marie-Henri Navier (1785-1836) comunicou à Academia de Paris, a primeira tentativa de um tratamento do equilíbrio e das vibrações em um continum elástico, marcando assim o nascimento da teoria matemática da elasticidade 23]. Navier considerou o meio como uma infinidade de partículas que agiam, umas sobre as outras, segundo as linhas que as une. Um distúrbio produzido sobre o meio elástico, então abandonado à ação de sua própria resiliência elástica era regido por uma equação que, em notação vetorial atual, pode ser escrita como

$$
\rho \frac{\partial^{2} \vec{e}}{\partial t^{2}}=\varepsilon[-\nabla \times(\nabla \times \vec{e})+3 \nabla(\nabla \cdot \vec{e})]
$$

a, hoje chamada, equação de Navier. Na expressão acima, $\rho$ é a densidade do meio material, $\vec{e}$ o vetor deformação, medida do quanto os pontos substanciais do meio estão deslocados relativamente à sua posição de equilíbrio, em um dado ponto do meio, e $\varepsilon$ (assim Navier o denotou) o parâmetro que define as suas propriedades elásticas.

Somente publicada em 1827, a mémoire de Navier levou Siméon-Dennis Poisson (1781-1840) [24] a considerar os modos independentes de propagação da onda de deformação elástica. Mostrou que se $\vec{e}$ é proveniente do diferencial exato de uma função $\phi$, isto é, como diríamos atualmente, se

$$
\vec{e}=\nabla \phi
$$

então de 10 resulta a equação de onda

$$
\frac{\rho}{3 \varepsilon} \frac{\partial^{2} \phi}{\partial t^{2}}-\nabla^{2} \phi=0
$$

a qual descreve um modo acústico (ou longitudinal), propagando-se com velocidade

$$
v_{l}=\sqrt{\frac{3 \varepsilon}{\rho}} .
$$

Alternativamente, se a deformação é puramente cisalhante (ou tranversal), o que corresponde à condição

$$
\nabla \cdot \vec{e}=0
$$

então 10 fornece

$$
\frac{\rho}{\varepsilon} \frac{\partial^{2} \vec{e}}{\partial t^{2}}-\nabla^{2} \vec{e}=0
$$

o que corresponde a uma onda propagando-se mais lentamente que a primeira, com velocidade

$$
v_{t}=\sqrt{\frac{\varepsilon}{\rho}}=\frac{v_{l}}{\sqrt{3}} .
$$

Poisson realizara, assim, de forma implícita, a decomposição do vetor deformação elástica, $\vec{e}$, em suas partes irrotacional (cf. (11)) e solenoidal (cf. (14)), o que mais tarde chamaremos decomposição de Helmholtz de um campo vetorial.

A contribuição mais fecunda surgida a partir da mémoire de Navier, no entanto, veio daquele que fora o revisor do seu artigo. Em uma sequência de artigos seminais 25 27. Luis Augustin Cauchy (1789-1857) estabeleceu os fundamentos para uma mecânica do contínuo elástico. No tocante a meios homogêneos e isotrópicos, Cauchy distinguiu duas formas de elasticidade, a primeira associada à resistência do meio à mudanças de forma, a qual associou o parâmetro elástico $k$. A segunda, a qual associou o parâmetro $K$, possibilitando uma resistência distinta à compressão/dilatação. Estendeu a Lei de Hooke ao contínuo elástico, através de uma equação constitutiva entre as pressões (ou tensões), como ele as chamava, e as deformações. Em notação indicial anacrônica escreveríamos

$$
T_{i j}=k e_{i j}+K \vartheta \delta_{i j}
$$

sendo

$$
\left\{T_{i j}\right\} \equiv\left\{T^{i j}\right\}=\left(\begin{array}{lll}
A & F & E \\
F & B & D \\
E & D & C
\end{array}\right)
$$

os componentes das pressões ou tensões, conforme denotados por Cauchy, atualmente o tensor de tensões,

$$
e_{i j}=\frac{1}{2}\left(\frac{\partial e_{i}}{\partial x^{j}}+\frac{\partial e_{j}}{\partial x^{i}}\right)
$$

o tensor deformação e

$$
\vartheta=\nabla \cdot \vec{e}
$$

o traço deste, que coincide com a divergência do vetor deformação $\vec{e}$. Os

$$
\left\{e_{i}\right\} \equiv\left\{e^{i}\right\}=(\xi, \eta, \zeta)
$$


conforme denotados por Cauchy, são os componentes cartesianos do vetor deformação.

As equações de movimento para as perturbações elásticas em um meio homogêneo e isotrópico, por ele obtidas, correspondem ao que podemos escrever, em notação indicial anacrônica, como

$$
\rho \frac{\partial^{2} e^{i}}{\partial t^{2}}=\frac{\partial T^{i j}}{\partial x^{j}},
$$

na qual usamos a convenção da soma de Einstein, isto é, de que há uma soma implícita onde há índices repetidos.

A partir da Lei de Hooke na forma (17), a dinâmica do campo de deformação 20 pode também ser escrita em notação vetorial, compactamente, como

$$
\rho \frac{\partial^{2} \vec{e}}{\partial t^{2}}=-\frac{k}{2} \nabla \times(\nabla \times \vec{e})+(k+K) \nabla(\nabla \cdot \vec{e}) .
$$

Esta equação de onda para as perturbações elásticas admite igualmente uma decomposição em modos acústico e cisalhante, cada qual com sua velocidade característica $\left(v_{l}, v_{t}\right)$, porém não mais obedecendo à razão de Poisson (16), graças à presença das duas constantes elásticas independentes $(k, K)$. Assim ocorria agora que

$$
v_{l}=\sqrt{\frac{k}{2 \rho}}, \quad v_{t}=\sqrt{\frac{k+K}{\rho}} .
$$

Os resultados de Navier podiam então ser recuperados admitindo-se que no meio em questão

$$
k=2 K=2 \varepsilon .
$$

Cauchy também considerou o problema da origem da elasticidade partindo de conjecturas sobre a constituição "molecular" do meio e sobre as forças de coesão entre elas, analogamente ao que fizera originalmente Navier, porém numa abordagem de maior generalidade, o que o habilitava a englobar substâncias cristalinas em seu esquema formal. Mostrou que haviam em geral 21 parâmetros elásticos distintos 27, necessários à caracterização das propriedades elásticas de sólidos não homogêneos e anisotrópicos, naquilo que virá a ser o embrião do nosso tensor de elasticidade atual. Conforme dito anteriormente, a discussão de tais aspectos foge ao escopo deste artigo.

\section{O éter luminífero na obra de Cauchy}

Em 1830 Cauchy aplicou sua teoria elástica em meios anisotrópicos aos problemas da óptica cristalina [28], isto é, supôs que o meio elástico em questão era o éter luminífero, naquela que ficou conhecida como a "Primeira teoria de Cauchy". Como decorrência desta, publicou no mesmo ano sua primeira teoria da reflexão, de um feixe polarizado em meios homogêneos e isotrópicos 29. Admitiu, em conformidade com sua abordagem em meios anisotrópicos, e diferentemente de Fresnel, que as vibrações luminais $\left(\vec{e}_{p}\right)$, associadas a cada modo de polarização, ocorriam nos correspondentes planos de polarização.
Como Fresnel, admitiu que as mesmas eram transversais à direção de propagação $(\hat{u})$. Admitiu também que a densidade do éter $(\rho)$ era a mesma em todos os meios e que eram as propriedades elásticas do meio luminífero $(k, K)$ que variavam de um meio transparente a outro. Assim, a diferença de velocidade de propagação da luz em meios transparentes distintos devia-se às variações nas propriedades elásticas. Matematicamente, se $\hat{n}_{p}$ é o vetor normal ao plano de polarização, então as premissas da primeira teoria da reflexão de Cauchy são:

$$
\vec{e}_{p} \propto \hat{u} \times \hat{n}_{p}, \quad \rho^{\prime}=\rho, \quad\left(k^{\prime}, K^{\prime}\right) \neq(k, K) .
$$

Assumindo então que o feixe de luz incidente estava polarizado perpendicularmente ao plano de incidência, obteve a lei dos senos (1) impondo como condição de contorno sobre a interface, $\Sigma$, a qual foi tomada como $x=0$, a continuidade da pressão normal à interface $\left(T_{x x}\right)$, bem como a continuidade do componente normal da velocidade $(\partial \vec{e} / \partial t)$. Isto é equivalente à

$$
\left[\frac{\partial e_{x}}{\partial t}\right]_{\Sigma}=0, \quad\left[T_{x x}\right] \propto\left[\frac{\partial e_{x}}{\partial x}\right]_{\Sigma}=0,
$$

Alternativamente, para um feixe incidente luz polarizado no próprio plano de incidência, impôs que as tensões cisalhantes produzidas sobre a interface eram contínuas, isto é,

$$
\left[T_{y z}\right]_{\Sigma} \propto\left[\frac{\partial e_{z}}{\partial y}\right]_{\Sigma}=0 \quad\left[T_{x z}\right]_{\Sigma} \propto\left[\frac{\partial e_{z}}{\partial x}\right]_{\Sigma}=0
$$

daí resultando a lei das tangentes (2).

Seus resultados surgiam assim invertidos, aparecendo a lei dos senos onde deveria surgir a das tangentes e reciprocamente; o que o levará, em anos posteriores, a reconsiderar toda sua óptica desde o começo.

Tais condições de contorno, em número de quatro, cumpriam exclusivamente a função de conduzirem às leis de Fresnel, num problema matematicamente bem posto, embora carecessem de fundamentação dinâmica.

No tocante aos modos longitudinais, sua ausência também carecia de justificativa física. Na medida em que as quatro condições de contorno podiam ser satisfeitas sem lançar mão dos mesmos, não havia necessidade matemática de introduzi-los. Assim, a ausência dos modos longitudinais carecia das mesmas justificativas físicas que as condições de contorno por ele usadas. Faltava-lhe, a rigor, uma fundamentação dinâmica.

Neste mesmo ano de 1830 a vida pessoal de Cauchy sofreu uma reviravolta, em decorrência da chamada revolução dos "Três dias gloriosos", que depôs o rei Charles $\mathrm{X}$. Cauchy sempre fora um "royaliste" e o fim da breve dinastia Bourbon, iniciada após a queda de Napoleão, o fez deixar Paris, levando consigo sua família, somente retornando a Paris em 1838 [30]. Durante seu exílio autoimposto Cauchy elaborou uma nova teoria da óptica cristalina 31,32, alternativa a de 1830, a qual ficou conhecida como "Segunda teoria de Cauchy", em que agora 
se reaproximava das escolhas originalmente assumidas por Fresnel, de que as vibrações da luz polarizada $\left(\vec{e}_{p}\right)$ ocorrem perpendicularmente ao plano de polarização, de que as diferenças de velocidade em diferentes meios eram devidas às mudanças de densidade $(\rho)$ e não das propriedades elásticas $(k, K)$. Matematicamente,

$$
\vec{e}_{p} \propto \hat{n}_{p}, \quad \rho^{\prime} \neq \rho, \quad\left(k^{\prime}, K^{\prime}\right)=(k, K) .
$$

Em 1836 apresentou sua segunda teoria da reflexão em meios isotrópicos 33, dentro do espírito de sua segunda teoria da óptica cristalina, na qual obteve as leis de Fresnel, conforme o caso, a partir de novas condições de contorno, a saber,

$$
[\nabla \times \vec{e}]_{\Sigma}=0, \quad\left[\frac{\partial e_{n}}{\partial n}\right]_{\Sigma}=0 .
$$

Novamente em número de quatro, estas garantiam que o problema matemático estava bem posto, mas a fundamentação física de tais condições de contorno permanecia ausente.

Em seu retorno a Paris publicou, em 1839, uma terceira teoria da reflexão [34, 35], iniciando a abordagem que adotará em suas publicações sobre óptica na década de 1840: a que ficará conhecida como do éter lábil ou contrátil de Cauchy. Sobre aquilo que já fizera neste campo de investigação ( 34 , p.677) admitiu que

"De resto, as pesquisas que publiquei nas Mémoires de l'Acadeémie e nos Exercices de Mathématiques [...] permanecem insuficientes para dar solução ao importante problema da reflexão e da refração dos raios luminosos."

Dois anos antes, George Green - de que falaremos adiante - afirmara que as ondas acústicas (longitudinais) deviam ser evitadas pela suposição de que fossem infinitamente rápidas, mas não pela suposição de que fossem infinitamente lentas. Green havia descartado a segunda opção por isto implicar num meio cujo equilíbrio seria instável (em termos atuais, isso significa tratar-se de um meio cujo módulo de incompressibilidade tem valor negativo). Em sua terceira teoria da reflexão, de 1839, Cauchy abraçou a segunda suposição.

Abordando o problema da onda incidente sobre a interface de dois meios homogêneos e isotrópicos dependente de duas constantes elásticas, às quais agora chamou $(i, f)$, em uma abordagem complexa, com todas as fases tomadas a princípio arbitrárias, o que lhe permite discutir o problema com grande generalidade, obteve as velocidades dos modos acústico e transversal em termos de suas novas constantes como

$$
c_{l}=\sqrt{i(1+f)}, \quad c_{t}=\sqrt{i} .
$$

A condição de que o meio fosse um sólido de NavierPoisson, era agora caracterizada por $f=2$. A de que fosse um meio que não propaga distúrbios longitudinais como $f=-1$. Em termos das constantes elásticas $(k, K)$ introduzidas por Cauchy em trabalhos anteriores, a condição de não propagação dos modos longitudinais seria $k=-K$.
Neste contexto demonstrou [36] as leis dos senos e das tangentes, (3) e (4), adotando, como em sua segunda teoria da reflexão, que

$$
\vec{e}_{p} \propto \hat{n}_{p}, \quad \rho^{\prime} \neq \rho,
$$

porém, no tocante às propriedades elásticas

$$
\left(k^{\prime}=-K^{\prime}, K^{\prime}\right)=(k=-K, K),
$$

Ainda, como condição de contorno adotou a continuidade da deformação elástica, através da interface, e a continuidade da taxa de variação desta na direção normal à interface. Matematicamente

$$
[\vec{e}]_{\Sigma}=0, \quad\left[\frac{\partial \vec{e}}{\partial n}\right]_{\Sigma}=0 .
$$

A primeira condição de contorno, usada por Cauchy pela primeira vez em sua obra, foi provavelmente herdada do trabalho de Green. A segunda, novamente, arbitrária e sem apelo físico imediato. Muito embora Cauchy ainda vá dedicar tempo e esforço à sua terceira teoria, no decorrer da década que adentrava, não resolverá afinal o problema do estabelecimento das condições de contorno fisicamente corretas sobre a interface.

Em síntese, podemos dizer que a ênfase de Cauchy sempre esteve centrada na consistência matemática e analítica da dedução das leis de Fresnel, entendidas como resultados exatos; diferentemente de autores como o próprio Fresnel ou, adiante, Green que buscaram uma abordagem mais fenomenológica e fundamentada na dinâmica.

Os permanentes reveses políticos na França pósnapoleônica parecem ter atenuado o protagonismo científico antes exercido pela França, e novas escolas científicas emergiam na Europa. Em Königsberg, na Alemanha, Franz Ernst Neumann (1798-1895) iria fundar uma escola de física matemática, a qual, juntamente com outras iniciativas e políticas de reforma universitária, descritos com grande maestria em [37], dará à Alemanha a liderança de que irá gozar ao final do século, produzindo figuras como Kirchhoff, Clausius, Helmholtz, Röntgen, Hertz e Planck. Neumann também produziu, independentemente de Cauchy, sua teoria da óptica baseada em no éter do tipo solido-elástico, que muito embora tenha estabelecido a continuidade da deformação através da interface também excluiu os modos longitudinais. Tais trabalhos somente seriam publicados em 1837 ( [5], p. 137.). Porém, queremos voltar nossa atenção à outra importante escola científica que irá assumir papel relevante na teoria da luz: a britânica.

\section{O éter tipo-geleia de Green}

George Green (1793-1841) viria a ser o precursor de uma nova geração de teóricos britânicos a revitalizar o protagonismo da velha universidade de Cambridge, entre os quais o jovem William Thomson (1824-1907), o futuro Lorde Kelvin, George Gabriel Stokes (1819-1903), George Bidel Airy. 
Em suas investigações no campo da óptica, Green adotou a mecânica de Lagrange como fundamento para estabelecer condições de contorno fisicamente aceitáveis, sobre a interface, harmonizando-as com a mecânica do meio elástico. Em seu "Sobre as leis da reflexão e refração da luz na interface comum de dois meios não-cristalinos" 38] de 1837, Green recolocou o problema enfrentado por Cauchy a partir de uma abordagem baseada nos métodos de Lagrange, segundo nos diz ele mesmo, conforme visto no Mécanique Analytique. Evitou considerações sobre a natureza microscópica do meio elástico, de que fosse atomística ou infinitamente divisível, bem como considerações sobre as interações aí escondidas, admitindo apenas que a soma efetiva de todas estas interações admitia um potencial. Introduziu assim o potencial elástico $(\mathcal{U})$ como uma função homogênea de grau dois das deformações. Esta depende, em geral, de 21 parâmetros arbitrários, que caracterizam as propriedades elásticas do meio. Em notação anacrônica o potencial elástico, introduzido por Green, se escreve como

$$
\mathcal{U}=\frac{1}{2} \mathcal{C}_{i j k l} e^{i j} e^{k l},
$$

sendo

$$
\mathcal{C}_{i j k l}=\mathcal{C}_{(i j)(k l)}=\mathcal{C}_{(i j k l)},
$$

os coeficientes que hoje identificamos como os componentes do tensor de elasticidade.

A densidade lagrangeana proposta por Green, em 1837, pode então ser caracterizada por

$$
\mathcal{L}_{\text {green }}=\frac{\rho}{2}\left(\frac{\partial \vec{e}}{\partial t}\right)^{2}-\mathcal{U} .
$$

Considerações de simetria o permitiram reduzir ainda mais o número de coeficientes elásticos (os graus de liberdade independentes) presentes em $\mathcal{C}_{i j k l}$. Em um meio homogêneo e isotrópico esse número ficou reduzido a apenas duas constantes, às quais foram designadas por $(A, B)$, de modo que poderíamos escrever em linguagem anacrônica

$$
\mathcal{C}_{i j k l}=B\left(\delta_{i k} \delta_{j l}+\delta_{i l} \delta_{j k}\right)+(A-2 B) \delta_{i j} \delta_{k l} .
$$

A imposição de que a dinâmica do sistema deve atender ao princípio variacional tanto nos semi-espaços cuja fronteira comum é $\Sigma$ quanto no espaço todo, forneceu naturalmente as condições de contorno a serem impostas sobre a interface comum dos dois meios, a saber, a da continuidade do vetor deformação $(\vec{e})$ e do vetor de tensão $\left(T^{i j} n_{j}\right)$ sobre a interface. Matematicamente,

$$
\left[e^{i}\right]_{\Sigma}=0, \quad\left[T^{i j} n_{j}\right]_{\Sigma}=0
$$

sendo $n_{j}$ os componentes do vetor normal $(\hat{n})$ à interface $\Sigma$. Estabelecia assim condições de contorno plenamente satisfatórias à luz dos princípios da mecânica. Como consequência, impunha-se a re-introdução de modos acústicos, ou longitudinais, coexistindo com os modos cisalhantes (a luz).
A dinâmica das deformações elásticas, por sua vez, em consonância com o princípio variacional, era dada por

$$
\rho \frac{\partial^{2} \vec{e}}{\partial t^{2}}=-B \nabla \times(\nabla \times \vec{e})+A \nabla(\nabla \cdot \vec{e})
$$

As velocidades de propagação das ondas acústicas e cisalhantes, explicitadas em termos das constantes introduzidas por Green, eram, respectivamente,

$$
c_{l}=\sqrt{\frac{A}{\rho}}, \quad c_{t}=\sqrt{\frac{B}{\rho}} .
$$

A correspondência com as constantes elásticas $(k, K)$, introduzidas anteriormente por Cauchy, é imediata, em conformidade com 21):

$$
B=\frac{k}{2}, \quad A=k+K
$$

Seguindo Fresnel, Green admitiu que as vibrações etéreas associadas à luz polarizada se dão perpendicularmente ao plano de polarização, conforme (5), e que as mudanças de velocidade de propagação em diferentes meios se devem às diferenças nas densidades dos meios.

No caso em que o plano de incidência coincide com o plano de polarização, a lei dos senos (1) foi demonstrada exatamente, sendo que o vetor deformação é tangencial à interface e, neste caso, a questão dos modos longitudinais sequer está colocada.

No caso em que o plano de polarização é perpendicular ao plano de incidência, o vetor deformação possui tanto componentes tangencial, quanto normal à interface. Nestas circunstâncias, as condições de contorno (37) somente podem ser satisfeitas se modos acústicos (longitudinais) forem gerados na interface; mesmo admitindo que não estavam presentes na onda incidente. Seguindo a ideia original de Fresnel, Green assumiu então que tais modos acústicos se propagavam infinitamente mais rápido que os modos cisalhantes (a luz), o que equivale à condição

$$
\frac{A}{B}=\left(\frac{c_{l}}{c_{t}}\right)^{2} \rightarrow \infty .
$$

Tal condição não gera o equilíbrio perpétuo da pressão, antes especulado por Fresnel, mas possibilita que os modos longitudinais formem ondas de superfície, que são evanescentes a partir da interface entre os dois meios, não se propagando no espaço ao redor. A consequência disto, porém, é que a razão das intensidades relativas não é propriamente a lei das tangentes (2). Trata-se de a uma relação mais complexa, da qual a lei das tangentes surge, como primeira aproximação. Green encerrou suas considerações tentando por em dúvida a validade exata da lei das tangentes, favorecendo a relação por ele obtida, o que a evidência experimental não corroborou; conforme 39

Green estendeu sua abordagem aos meios cristalinos em 1839 40], em artigo lembrado por Whittaker [5] como "memorável" e por Sokolnikoff [41] como "revolucionário". 
Continha aí, na verdade, duas teorias que se assemelhavam às primeira e segunda teorias de Cauchy. Na primeira se admite que as tensões no éter não perturbado são nulas e que as vibrações etéreas associadas à luz polarizada estão no plano de polarização, conforme (24). Na outra a tensão inicial é não nula e as vibrações da luz polarizada são perpendiculares ao plano de polarização, como em (5). Ficaram conhecidas como as primeira e segunda teorias de Green.

Nas décadas seguintes aos trabalhos de Green o éter do tipo-geleia dominou a cena, muito embora sem ser capaz de reproduzir exatamente a lei de Fresnel das tangentes, na reflexão em meios isotrópicos. George Gabriel Stokes agregou a ele certa viscosidade, dando origem às teorias de um éter visco-elástico 42,43. A questão do estado de movimento do meio etéreo diante do movimento dos corpos ponderáveis estivera presente no debate desde Fresnel, que defendera que um corpo em movimento põe o éter parcialmente em movimento consigo, o chamado arrasto do éter. Stokes introduziu a viscosidade com o intuito de conciliar a necessidade do éter ser rígido para distúrbios rápidos, como aqueles associados à luz, e virtualmente indiferente a distúrbios lentos, como os associados ao movimento planetário. Esta viscosidade o tornava estacionário relativamente à Terra, na vizinhança imediata da Terra, o que seus contemporâneos julgavam ser uma dificuldade frente ao fenômeno da aberração estelar. Stokes mostrou que, contrariamente, a premissa do éter localmente estacionário não contradizia as observações da aberração estelar, pois os efeitos se cancelavam em ordem de $v / c$. O argumento não se aplica à segunda ordem $\left(v^{2} / c^{2}\right)$ e muito embora este não tenha sido um problema à época, sabemos retrospectivamente que mais tarde se tornará dramático, estando a não detecção do arrasto do éter, em quaisquer experiências, ligada à origem da Relatividade Especial.

Fugindo à regra do éter visco-elástico mencionamos o caso do éter girostático de James MacCullagh (18091847) 44, publicado em 1848, no qual se assume que o meio luminífero somente resistiria à rotação $(\nabla \times \vec{e})$, não à compressão/descompressão nem à deformação. Em notação anacrônica podemos dizer que a densidade lagrangeana associada ao meio girostático seria

$$
\mathcal{L}_{m a c}=\frac{\rho}{2}\left(\frac{\partial \vec{e}}{\partial t}\right)^{2}-\frac{\bar{\mu}}{2}(\nabla \times \vec{e})^{2}
$$

em que $\rho$ é a densidade do meio e $\bar{\mu}$ a rigidez girostática, do meio. No éter de MacCullagh se pode deduzir as leis de Fresnel, (1) e 22, de forma exata. No entanto, o tipo de "elasticidade" puramente rotacional é estranha ao meios ponderáveis conhecidos. Além disto, embora MacCullagh adotasse a continuidade de $\vec{e}$ através da interface, a outra condição de contorno carecia de apelo físico: tratava-se da continuidade do componente tangencial da "tensão girostática" $\bar{\mu} \nabla \times \vec{e}$, a qual denotamos por $\bar{\mu}(\nabla \times \vec{e})_{\|}$. Assim, as condições de contorno podem ser escritas como

$$
[\vec{e}]_{\Sigma}=0, \quad\left[\bar{\mu}(\nabla \times \vec{e})_{\|}\right]_{\Sigma}=0
$$

James MacCullagh não foi um ardoroso defensor das qualidades inerentes ao "éter girostático" tanto na óptica dos meios isotrópicos como também cristalinos. Foi, pouco depois, desqualificado por Stokes em 1862 [45] como meio insustentável pelos princípios da mecânica, pois, para dizermos de forma breve, significaria associar as tensões do meio luminífero a um tensor de tensões antissimétrico. Tal antissimetria impede que um estado de equilíbrio rotacional possa se estabelecer em uma porção do meio. Nenhum meio conhecido apresentava rigidez girostática.

\section{A teoria eletromagnética é o fundamento?}

Também nas décadas seguintes, James Clerk Maxwell (1831-1879) construiu a teoria do campo eletromagnético, que era, então, um programa de unificação dos "éteres" luminífero e eletromagnético, isto é, um esforço no sentido de se entender tanto os fenômenos eletromagnéticos quanto os de radiação (aí incluída evidentemente a luz) como ações resultantes dos estados de deformação e movimento de um só meio etéreo. Quando em 1862 Maxwell introduziu sua ideia-chave da corrente de deslocamento [46], claramente remetia todas as variáveis de campo às propriedades mecânicas de um éter único, pensado como um "mar de vórtices moleculares." Aí postulou uma correspondência do campo magnético $(\vec{H})$ com o movimento turbilhonar no interior do vórtice molecular, bem como do vetor deslocamento elétrico $(\vec{D})$ com a deformação elástica de tais moléculas etéreas. Maxwell assumiu ainda que a elasticidade do meio era compatível à de um sólido de Navier-Poisson, o que implicava que a velocidade das ondas cisalhantes no "éter livre" coincidiam com a velocidade da luz. Assim se expressou em oração tão repetida desde então ( [46], p.22):

"A velocidade das ondulações transversais em nosso meio hipotético [...] concorda tão exatamente com a velocidade da luz [...] que dificilmente podemos evitar a inferência de que a luz consiste nas ondulações transversais do mesmo meio que é a causa dos fenômenos elétricos e magnéticos."

Porém, em se tratando de um sólido de Navier-Poisson, aí subsistia o problema dos modos acústicos, movendo-se mais rapidamente que a luz por um fator de $\sqrt{3}$, como em (16). Assim, uma teoria da reflexão moldada pela teoria de Maxwell original sofreria, fatalmente, dos mesmos males, e até piores, que as teorias do tipo-geleia. Mesmo em se tratando de um trabalho em progresso, conforme Hunt ( [52], p.77) este era, provavelmente, o artigo de Maxwell mais apreciado pelos leitores contemporâneos de sua obra eletromagnética; na medida em que havia o claro esforço em revelar os elos mecânicos entre as variáveis de campo e a cinemática e/ou dinâmica do meio. A partir de 1864 Maxwell irá se afastar deste compromisso explícito com a mecânica do éter 47 defendendo a primazia de suas equações do campo eletromagnético na 
descrição dos fenômenos eletromagnéticos e ópticos, sem estabelecer a correlação explícita entre as variáveis de campo e as correspondentes variáveis mecânicas do meio etéreo. A isso apelidamos no início deste trabalho de "a apropriação" da luz: a luz é antes uma onda eletromagnética, que uma onda de deformação cisalhante do éter. E isto é condição necessária e suficiente para eliminar os modos longitudinais no espaço livre (isto é, no éter livre).

Sob a perspectiva da física do plenum o programa da teoria eletromagnética permanecia inacabado, com a morte prematura de Maxwell, em 1879, haja visto que a correlação entre as variáveis de campo e as características mecânicas do meio jaziam ocultas. Foi George Francis FitzGerald (1851-1901) quem retomou a teoria da reflexão/refração no contexto da teoria eletromagnética, em 1880 [48]. Estabeleceu uma correspondência entre os éteres de Maxwell e o éter girostático de MacCullagh, o qual era capaz de fornecer as leis de Fresnel. Para tornar efetiva tal correspondência fazia-se necessário associar as variáveis de campo $\vec{H}$ e $\vec{D}$ à deformação elástica rotacional $\vec{e}$, de MacCullagh, como

$$
\vec{H}=\frac{\partial \vec{e}}{\partial t}, \quad \vec{D}=\nabla \times \vec{e} .
$$

A essa altura, contudo, Maxwell já havia depurado seu legado eletromagnético na forma de um tratado de eletricidade e magnetismo. No "Treatise" de Maxwell 49 encontrava-se que

$$
\vec{H}=\frac{1}{\mu} \nabla \times \vec{A}, \quad \vec{D}=-\epsilon \frac{\partial \vec{A}}{\partial t},
$$

sendo $\mu$ a permeabilidade magnética do meio e $\vec{A}$ a função que representava o estado eletrotônico, também chamada, por Maxwell de momento eletromagnético. Na nomenclatura atual, o potencial vetorial magnético.

Assim, apesar do avanço de FitzGerald visando consolidar a teoria eletromagnética nos domínios da óptica, a correlação exata entre as variáveis que descrevem o campo eletromagnético no éter livre $(\mu, \epsilon, \vec{A})$ e as características do éter subjascente,mantinha-se oculta e ambígua; fosse ele o éter de MacCullagh $(\rho, \bar{\mu}, \vec{e})$ ou fosse ele o de Green $(\rho, A, B, \vec{e})$.

\section{Thomson: a elasticidade como fundamento}

Muito embora a produção de ondas eletromagnéticas em laboratório marque o ponto de inflexão para a ampla aceitação da teoria eletromagnética de Maxwell, haviam, ainda assim, opiniões discordantes de relevo.

William Thomson fora uma espécie de mentor do jovem Maxwell nos seus primeiros ensaios teóricos visando dar sentido matemático preciso às linhas de força de Michael Faraday. No entanto afastara-se da abordagem eletromagnética de Maxwell, à medida em que este se descomprometia mais e mais em formar uma imagem mecânica bem definida de suas variáveis de campo e, consequentemente, de suas equações eletromagnéticas. Rejeitou a "assim chamada teoria eletromagnética da luz", em suas Baltimore Lectures [50] (p. 9), em 1884, assim se manifestando:

"Se eu soubesse o que é a teoria eletromagnética da luz, eu poderia pensar nela em relação aos princípios fundamentais da teoria ondulatória da luz. Mas ela antes parece a mim como um passo para trás de uma noção mecânica absolutamente definida [...]."

Mesmo na versão revisada de suas Baltimore Lectures, de 1904, Thomson manterá a posição sustentada acima.

Em que pese o que Hertz chamou de "apelo superior" da teoria de Maxwell, por se tratar de um programa de unificação, nem todos viam razões para aceitar as equações do campo eletromagnético como leis físicas fundamentais. Como consequência, não necessariamente se prestavam à ser o fundamento dos fenômenos ópticos. Thomson esteve entre os que mantiveram uma atitude de fundamentar a óptica diretamente na mecânica do meio etéreo, sem subjugar a luz ao campo eletromagnético, muito embora fosse ele, paradoxalmente, um dos fundadores do programa de unificação dos éteres.

Em 1888, o encontro da British Association for the Advancement of Science (BAAS) deu-se sob o calor dos feitos de Hertz, que produzira ondas eletromagnéticas em laboratório; em Karlsruhe-Alemanha. Podemos ter uma ideia da sensação que isto causou, no seio da comunidade britânica, pela tônica dada por FitzGerald, em sua fala de abertura da seção de física e matemática, em 6 de setembro [51] (p.561):

"Assim como as pesquisas de Young e Fresnel sobre a interferência da luz provaram a teoria da óptica ondulatória, assim os experimentos de Hertz provam a teoria etérea do eletromagnetismo."

É importante atentar às palavras aqui. No paradigma da física do plenum, a prova empírica da existência de forças eletromotrizes se propagando na forma de ondas, por Hertz, não confirmava a teoria eletromagnética de Maxwell. O que seguramente demonstrava era que o espaço está efetivamente preenchido por um meio, que este meio tem algum tipo de elasticidade e, por intermédio dela, que propaga não somente a luz mas também as ações eletromagnéticas.

Para o encontro da BAAS, em 1888, Thomson havia preparado uma comunicação intitulada "A Simple Hypothesis for Electro-Magnetic Induction of Incomplete Circuits", na qual insistia na abolição da corrente de deslocamento, deixando apenas correntes de condução. Conforme Hunt [52, o clima do encontro, à luz das descobertas de Hertz, bem como a presença de entusiastas do eletromagnetismo de Maxwell, como o próprio FitzGerald, levaram Thomson a uma momentânea relativização de suas opiniões, antes de sua apresentação, ocorrida no dia 7. Ele modificou sua caracterização da corrente de deslocamento da condição de hipótese "wholly untenable" (totalmente insustentável) para "not wholly tenable" 
(não totalmente sustentável). Tal atitude nos ajuda a compreender o porquê de Thomson decidir reavivar a memória de seus contemporâneos para a terceira teoria de Cauchy, em artigo publicado no Philosophical Magazine de novembro 39] daquele mesmo ano.

Referência entre seus pares na filosofia natural de seu tempo, Thomson publicara em 1883 o volume II do seu "Treatise on Natural Philosophy", escrito em parceria com Peter Guthrie Tait. Texto de referência em filosofia natural, nele se apresentava a teoria da elasticidade caracterizando as propriedades do meio elástico hookesiano, homogêneo e isotrópico, em termos do módulo de incompressibilidade, $\kappa$, e do módulo de rigidez, $n$, que constitui a forma padrão da Lei de Hooke ainda em uso em livros didáticos atuais. Em notação anacrônica a Lei de Hooke é aí apresentada na forma [53] (p. 693),

$$
T_{i j}=2 n e_{i j}+\left(\kappa-\frac{2}{3} n\right) \vartheta \delta_{i j} .
$$

Deste modo, as constantes elásticas introduzidas por Thomson \& Tait, relacionam-se às de seus antecessores por

$$
n=B=\frac{k}{2}, \quad \kappa=A-\frac{4}{3} B=K+\frac{1}{3} k .
$$

Aí também se encontra a formulação do princípio da conservação da energia no meio elástico, sendo demonstrado que o trabalho $(W)$ realizado para se modificar o estado de tensão de uma porção do meio elástico, contida em um volume $V$, desde a condição não deformada $(\vec{e}=$ $0)$ até aquela caracterizada por um campo de deformação, $\vec{e}$, é

$$
W=\int_{V} \frac{1}{2} e_{i j} T^{i j} d V .
$$

No mencionado artigo de novembro de 1888 Thomson retoma a ideia descartada por Green, de eliminar os modos longitudinais tomando-se a velocidade de propagação destes nula. Isso requer, na linguagem por ele introduzida em seu "Treatise" que o módulo de incompressibilidade $(\kappa)$ seja negativo-definido e dado em termos do módulo de rigidez $(n)$ por

$$
\kappa=-\frac{4 n}{3} .
$$

Para Thomson, a adoção de $\kappa<0$ é justificável, com base no princípio da conservação da energia. Lembra-nos ele que Green sequer possuía as palavras "energia" ou "trabalho". O argumento é o seguinte: se o meio, como um todo, for contido em um vaso rígido, isto é, uma fronteira sobre a qual a deformação se anula, então o trabalho realizado para deformá-lo depende das deformações puramente rotacional $(\nabla \times \vec{e})$ e puramente longitudinal $(\nabla \cdot \vec{e})$ do seguinte modo:

$$
W=\frac{1}{2} \int_{V}\left\{n(\nabla \times \vec{e})^{2}+\left(\kappa+\frac{4}{3} n\right)(\nabla \cdot \vec{e})^{2}\right\} d V .
$$

Assim, mesmo adotando (49), realiza-se trabalho motor para deformar o meio, em relação à sua condição não deformada, dado por

$$
W_{\text {labil }}=\frac{1}{2} \int_{V} n(\nabla \times \vec{e})^{2} d V,
$$

desde que $n>0$.

Assumindo tais condições ele então demonstra, no referido artigo, que num éter lábil se pode obter exatamente as leis de Fresnel, tanto a dos senos (1) quanto a das tangentes, (2), admitindo-se que as oscilações ocorrem perpendicularmente ao plano de polarização, conforme Fresnel, e tomando-se como condições de contorno sobre a interface de separação as mesmas de Green (37).

Foi Thomson quem nomeou o éter luminífero com a característica (49) de "éter contrátil" ou "lábil" de Cauchy e aquele introduzido por Green de "éter tipo geleia".

Em trabalhos posteriores Thomson também retomará o éter girostático de MacCullagh, propondo até mesmo sistemas mecânicos que apresentavam uma elasticidade quase-girostática [54].

\section{Conclusão}

A teoria ondulatória da luz baseada no éter tipo sólido elástico revelou-se afinal incapaz de fornecer uma explicação das leis de Fresnel de modo intuitivo e natural, isto é, sem dotar o meio de propriedades elásticas exóticas ou lançar mão de condições de contorno sem apelo mecânico. A presença dos modos longitudinais indesejados eram a característica marcante de qualquer modelo "aceitável" mecanicamente. Mesmo assim ela desempenhou papel significativo na concretização da ideia de que o "espaço livre" é o repositório de tensões que através dele se distribuem ou mesmo se propagam. Ela guiou a construção da teoria eletromagnética da luz fornecendo elementos-chave para a construção de um só éter óptico-eletro-magnético, o qual, no entanto, jamais foi postulado de forma plenamente consistente pelo próprio Maxwell. Nela se revelou a relevância das equações diferenciais parciais na descrição dos processos físicos, bem como a impossibilidade de se ter um problema de valor de contorno bem posto sem prescrever condições de contorno adequadas. Mesmo em nossos textos didáticos atuais de teoria eletromagnética, os teoremas de Green, relativos à problemas de valor de contorno, permanecem em destaque nos capítulos dedicados à fundamentação matemática. Em tais textos didáticos, no entanto, pouco se percebe o caráter coletivo da construção das hoje chamadas Equações do campo eletromagnético, atributo este tão frequentemente lembrado quando se trata da Mecânica Quântica.

Ao promover a "apropriação" da luz para dentro da dinâmica do campo eletromagnético, Maxwell deixava de lado o compromisso com a interpretação literal das variáveis que descrevem o comportamento da luz e os movimentos, tensões e deformações do éter. As condições de contorno podiam assim buscar justificativa nas leis do campo eletromagnético, sem lhes atribuir significado mecânico. Dentro do paradigma de uma física totalmente 
mecanicista perdeu clareza e substância, o que desagradava gente da estatura de Thomson, por exemplo. Aceitar a primazia das equações do campo eletromagnético, postuladas como primeiros princípios, era a opção reducionista. Isto implicava, aos olhos dos defensores da física do plenum, não numa síntese, como vemos hoje, mas numa renúncia

Não podemos deixar de lembrar que as virtudes e limitações da teoria elástica da luz na fundamentação das leis de Fresnel não são suficientes para fornecer um quadro completo das mesmas virtudes e limitações do próprio programa teórico de uma física do plenum. As questões relacionadas à dispersão da luz e à aberração estelar impuseram desafios os quais o programa do éter visco-elástico também ofereceu solução parcial engenhosa, mas que deram lugar, afinal, aos impasses na detecção do referencial de repouso local do éter. Tais temas somente poderíamos abordar apropriadamente em outra oportunidade.

\section{Referências}

[1] M. Heald e J.B. Marion, Classical Electromagnetic Radiation (Brooks/Cole, Boston, 1995), p. 207, $3^{\text {a }}$ ed.

[2] D.J. Griffiths, Introduction to Electrodynamics (Prenticehall Inc., New Jersey, 1999), p. 390, 392, $3^{\text {a }}$ ed.

[3] M. Born e E. Wolf, Principles of Optics (Cambridge University Press, Cambridge, 1999), p. 42, $7^{\text {a }}$ ed.

[4] R. Descartes, Princípios da Filosofia (Edições 70, Lisboa, 2006).

[5] E.T. Whittaker, A History of the Theories of Aether and Electricity: The classical theories (Thomas Nelson and Sons, London, 1951).

[6] R. Hooke, Micrographia or Some Physiological Description of Minute bodies (Cosimo Inc., New York, 2007).

[7] C. Huygens, Treatise on Light (MacMillan and Co., London, 1912).

[8] I.B. Cohen e R.S. Westfall, NEWTON - textos, antecedentes, comentários (Ed. Contraponto-UERJ, Rio de Janeiro, 2002), p. 39, p. 408.

[9] J. Bernoulli, Recherches physiques et géométriques sur la question: Comment se fait la propagation de la lumière (Imprimerie Royale, Paris, 1736).

[10] T. Young, Phil. Trans. R. Soc. Lond. 94, 1 (1804).

[11] D. Brewster, Phil Trans. R. Soc. Lond., 105, 125 (1815).

[12] D. Brewster, Phil. Trans. R. Soc. Lond., 105, 270 (1815).

[13] A. Fresnel, Mémoires de l'Académie Royale des Sciences v, 339 (1826).

[14] J.Z. Buchwald, The Rise of the wave theory of light (University Chicago Press, Chicago, 1989).

[15] I. Newton, Optiks (Dover Publ. Inc., New York, 1952), p. 363.

[16] D. Arago e A. Fresnel, Annales de Chimie et de Physique x, 288 (1819).

[17] A. Fresnel, Annales de Chimie xvii, 180 (1821).

[18] R.H. Silliman, in: Dicionário de Biografias Científicas, editado por César Benjamin (Contraponto Editora, Rio de Janeiro, 2007), p. 860.

[19] A. Fresnel, Annales de Chimie et de physique xvii, 102 (1821).
[20] H. Hertz, On Electric Radiation (Electric Waves, New York, 1962).

[21] A. Fresnel, Prémier mémoire sur la double réfraction Oeuvres (Imprimerie Impériale, Madison, 1868), p. 261.

[22] A. Fresnel, Mémoires de l'Academie Royale des Sciences xi, 393 (1832).

[23] M. Navier, Mém. de l'Acad. des Sciences vii, 375 (1827).

[24] S.D Poisson, Mémoire de l'Académie des Sciences viii, 623 (1828).

[25] A. Cauchy, Exercices de Mathématiques, iii, 160 (1828).

[26] A. Cauchy, Exercices de Mathématiques, iii, 188 (1828).

[27] A. Cauchy, Exercices de Mathématiques, iii, 213 (1828).

[28] A. Cauchy, Mémoire de l'Académie des Sciences x, 293 (1831).

[29] A. Cauchy, Bulletin des Sciences Mathématiques xiv, 6 (1830).

[30] H. Freudenthal, in: Dicionário de Biografias Científicas, editado por César Benjamin (Contraponto Editora, Rio de Janeiro, 2007), p. 422.

[31] A. Cauchy, Comptes Rendus ii, 341 (1836).

[32] A. Cauchy, Mémoire de l'Académie des Sciences xviii, 153 (1842).

[33] A. Cauchy, "Mémoire sur la dispersion de La lumière" (Société oyale des Sciences, Praga, 1836).

[34] A. Cauchy, Comptes Rendus ix, 676 (1839).

[35] A. Cauchy, Comptes Rendus ix, 726 (1839).

[36] A. Cauchy, Comptes Rendus x, 347 (1840).

[37] C. Jungnickel e R. McCormmach, Intellectual Mastery of Nature - Theoretical Physics from Ohm to Einstein (University Chicago Press, Chicago, 1986).

[38] G. Green, Trans. of the Camb. Phil. Soc., 7, 1 (1838).

[39] W. Thomson, Phil. Mag., 26, 414 (1888).

[40] G. Green, Trans. Camb. Phil. Soc., 7, 121 (1839).

[41] I. Sokolnikoff, Mathematical Theory of Elasticity (McGraw-Hill Book Company, New York, 1956).

[42] G.G. Stokes, Phil. Mag., 27, 9 (1845).

[43] G.G Stokes, Phil. Mag., 29, 6 (1846).

[44] J. MacCullagh, Transactions of Royal Irish Academy xxi, 17 (1848).

[45] G.G. Stokes, Report on Double Refraction (Taylor and Francis, Abingdon-on-Thames, 1862), p. 253.

[46] J.C. Maxwell, Philosophical Magazine 23, 12 (1862).

[47] J.C. Maxwell, The Scientific Papers of James Clerk Maxwell (Dover Publ. Inc., New York, 1965), p. 526.

[48] G.F. FitzGerald, Philosophical Transactions 171, 691 (1880).

[49] J.C. Maxwell, A Treatise on Electricity and Magnetism (Oxford University Press, Oxford, 2002).

[50] W. Thomson, Baltimore Lectures: Molecular Dynamics and the wave theory of Light Cambridge University Press, London, 1904).

[51] G.F. FitzGerald, in Report of the Fifty-Eighth Meeting of the British Association for the Advancement of Science (Spottiswoode and Co., London, 1889), p.557.

[52] B.J. Hunt, The Maxwellians (Cornell University Press, Ithaca, 1991).

[53] W. Thomson e P.G.Tait, Treatise on Natural Philosophy (Cambridge University Press, Cambridge, 1912).

[54] W. Thomson, Comptes Rendus 109, 453 (1889). 